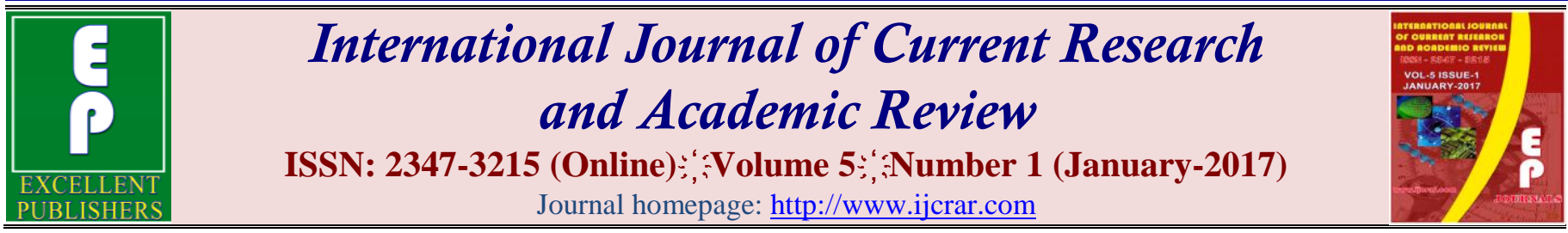

\title{
Estimation of Serum Total Bilirubin Level and Hyponatremic Effect Associated with the Use of Levetiracetam Therapy in Epileptic Patients
}

\author{
Lincy Joseph $^{1}$, Mathew George ${ }^{2}$ and Lintu Mariam Joshua ${ }^{2} *$ \\ ${ }^{1}$ Department of Pharmaceutical Chemistry, Pushpagiri College of Pharmacy, Thiruvalla-689107, Kerala, India \\ ${ }^{2}$ Department of Pharmacology, Pushpagiri College of Pharmac, Thiruvalla-689107, Kerala, India
}

*Corresponding author: lintujoshu93@gmail.com

\begin{abstract}
Epilepsy is a group of neurological diseases characterized by epileptic seizures. Levetiracetam is increasingly used as adjunctive anticonvulsant therapy. Levetiracetam is second generation anti epileptic drug. The present review is aimed on the estimation of serum total bilirubin level and hyponatremic effect associated with the use of levetiracetam therapy in epileptic patients. The clinical monitoring of the serum sodium levels is done to identify the development of hyponatremia associated with the use of levetiracetam. Asymptomatic elevation in serum total bilirubin is found in patients taking levetiracetam. The review provides clinical information about the incidence of hyponatremia and serum total bilirubin level associated with levetiracetam therapy.
\end{abstract}

\section{Article Info}

Accepted: 25 December 2016

Available Online: 10 January 2017

\section{Keywords}

Epileptic patients, levetiracetam, serum total bilirubin level,

hyponatremia, Clinical monitoring.

\section{Introduction}

A seizure is the manifestation of an abnormal, hypersynchronous discharge of a population of cortical neurons. Epilepsy is a group of disorder characterized by two or more unprovoked seizures. Epilepsy is classified based on the source of seizure into partial and generalized seizures. Epilepsy is a group of neurological diseases characterized by epileptic seizures. Epileptic seizures are episodes that can vary from brief and nearly undetectable to long periods of vigorous shaking (Zimmerman, 1999). Levetiracetam is second generation anti epileptic drug. Levetiracetam is available as tablets of 250, 500, 750 and $1000 \mathrm{mg}$ generically and under the brand name Keppra. Liquid oral and injectable forms are also available. The recommended initial dose in adults is $500 \mathrm{mg}$ twice daily with dose escalation based upon tolerance and effect to a maximum of 1500 mg twice daily. Dosing in children is based upon body weight. Common side effects include dizziness, somnolence and fatigue. The treatment of epilepsy depends on appropriate classification of seizure type and the epileptic syndrome The older or first generation antiepileptic drugs like phenytoin, carbamazepine and sodium valproate. Hyponatremia is defined as a decrease in the serum sodium concentration to a level below 136 mmolper liter.

Levetiracetam is approved for adjunctive therapy for adults with partial, myoclonic, and generalized tonic clonic seizures. Patients taking levetiracetam found to have hyponatremia and their serum total bilirubin level is also elevated. So patients taking levetiracetam should monitor sodium and serum total bilirubin level 
Hyponatremia is an important and common electrolyte abnormality that can be seen in isolation or, as most often is the case, as a complication of other medical illnesses. Symptoms range from nausea and malaise, with mild reduction in the serum sodium, to lethargy, decreased level of consciousness, headache, and (if severe) seizures and coma. Overt neurologic symptoms most often are due to very low serum sodium levels (usually $<115 \mathrm{mEq} / \mathrm{L}$ ), resulting in intracerebral osmotic fluid shifts and brain edema (Neligan et al., 2012).

Hypoosmolality (serum osmolality $<280 \mathrm{mOsm} / \mathrm{kg}$ ) always indicates excess total body water relative to body solutes or excess water relative to solute in the extracellular fluid (ECF), as water moves freely between the intracellular and the extracellular compartments. This imbalance can be due to solute depletion, solute dilution, or a combination of both.Thus, hyponatremia can occur only when some condition impairs normal free water excretion. Therefore, correction of hyponatremia must take into account the chronicity of the condition. Acute hyponatremia (duration $<48 \mathrm{~h}$ ) can be safely corrected more quickly than chronic hyponatremia. Correction of serum sodium that is too rapid can precipitate severe neurologic complications. Most individuals who present for diagnosis, versus individuals who develop it while in an inpatient setting, have had hyponatremia for some time, so the condition is chronic, and correction should proceed accordingly (Neligan et al., 2012).

\section{Review of Literature}

Meshkini et al., (2015) conducted a study based on "Comparison of levetiracetam versus phenytoin for seizure prophylaxis in patients with traumatic brain injury". Phenytoin (PHT) is used for seizure prophylaxis in patients with traumatic brain injury (TBI). However, levetiracetam (LEV) is emerging as an alternative. Hence in this study, we aimed to conduct a meta-analysis comparing these two drugs in patients with TBI. A systematic search in electronic databases was performed. Studies consistent with purpose (comparing LEV vs. PHT for the prevention of seizures in TBI patients) were selected for our meta-analysis. They extracted data of all eligible studies on a standard abstraction sheets. Extracted data included patient's demographics, study type, intervention, and outcome. We defined seizures as primary outcome. 1184 unduplicated papers identified by our search of which 1106 were excluded by reading the abstract and titles. 72 papers were removed by reading the full text. Finally 6 studies (Cohort studies) were selected for analysis. There is no superiority of either these two drugs at preventing of seizures based on the point estimate's odds ratio $(\mathrm{OR})=1.1$ [95\% confidence interval $(\mathrm{CI})=0.55-2.20]$. The result of the study shows that PHT and LEV showed equal efficacy in prevention of seizures after TBI.

Swaroop et al., (2013); conducted a study based on Levetiracetam: A Review of its use in the treatment of epilepsy. Levetiracetam is a novel antiepileptic drug. It is marketed worldwide since 2000. The aim of the study is to review the mechanism of action, pharmacokinetics, adverse drug reactions, contraindications and uses of levetiracetam in the treatment of various types of epileptic seizures. The study shows that Levetiracetam acts by binding to the synaptic vesicle protein SV2A, thereby modulation of one or more of its actions and ultimately affecting neural excitability. It has less protein binding and lacks hepatic metabolism. In contrast to traditional therapy, it has a wide safety margin and does not require serum drug monitoring. It does not interact with other anti-epileptics. The study shows that pharmacological benefits of levetiracetam make it an important first-line or adjunctive therapy for epileptic seizures.

Hae Won Shin et al., (2013) conducted a study based on "Review of Levetiracetam as a First Line Treatment in Status Epilepticus in the Adult Patients". With the advent of new antiepileptic drugs comes the potential for significant advances in the emergent management of status epilepticus. Traditional antiepileptic drugs possess side effect profiles that may limit their clinical utility or lead to increased patient morbidity or mortality. The relatively recent development of levetiracetam shows promise for effective control of acute status epilepticus in adults, but current objective data of its use as a firstline agent for control of status is quite limited. This study serves to examine existing literature while considering levetiracetam as a first-line therapy in status in the adult patient population. Although existing studies are narrow in their scope, the present data lay a substantial foundation for further investigation of levetiracetam as a primary therapy in acute status epilepticus. Studies indicate that levetiracetam is an attractive option in patients who are unable to be treated with traditional first-line therapies. This may serve as a catalyst for the exploration of levetiracetam as a first-line medication.

Aasim Ali Syed et al., (2012) conducted a study on Acute liver failure following levetiracetam therapy for seizure prophylaxis in traumatic brain injury". This case report investigates the occurrence of drug induced acute 
liver injury directly associated with the administration of levetiracetam in a patient following traumatic brain injury. Levetiracetam (Keppra) is an anti-epileptic drug commonly used as an adjunct treatment for partial-onset seizures. It's mechanism of action involves blocking Ntype calcium channels and indirectly inducing GABA and glycine pathways resulting in the blunting of excessive neuronal activity. Levetiracetam is absorbed completely into the blood, which yields a high bioavailability. However, the drug does not bind to plasma proteins. In patients with severe liver cirrhosis, total clearance was reduced by $57 \%$ and a reduction in dose by $50 \%$ is recommended. The adverse events most commonly associated with the use of levetiracetam are behavioral or psychological disturbances (33\%) and fatigue and somnolence (33\%). The results shows that After the administration of of the levetiracetam, there was rapid elevation in liver enzymes and levetiracetam was discontinued 4 days later.

Vincenzo Belcastro et al., (2008) conducted a study on "Levetiracetam-associated hyponatremia".The earlier studies shows that oxcarbazepine, and occasionally with valproate and lamotrigine causes hyponatremic condition. Upto this date,there is only a report of hyponatremia associated with levetiracetam in a 65-yearold patient predisposed to the development of syndrome of inappropriate antidiuretic hormone secretion (SIADH).We recently observed a patient developing hyponatremia following two challenges with levetiracetam in absence of any evident pre-disposition to SIADH.Patient developed low serum sodium levels at different levetiracetam doses (i.e., 500 and $1000 \mathrm{mg}$ twice daily), according to the absence of association between the AED dose and hyponatremia.Thus, subjects taking levetiracetam and presenting these risk factors should be particularly monitored for laboratory evidence of hyponatremia.

Gordon Gibson et al., (2002) conducted a study based on the "Efficacy of levetiracetam in developmentally disabled patients". Objective of the study was to investigate whether seizures in developmentally disabled are often refractory to treatment. This study aimed to expand the clinical experience with levetiracetam as an antiepileptic drug (AED. Methods include four males and two females (aged 25-51) with mental retardation requiring institutionalization and uncontrolled seizures (1.7-12.2/month) received levetiracetam (0.5-2.5g/day) in addition to their standard AEDs. Clinical response was closely followed for 4-10 months (mean, 6 months) with respect to seizure control and adverse effects. The result shows that two of the patients became seizure-free, while the other four had seizure reductions ranging from 71 to $92 \%$. None of the patients experienced adverse effects, and three had improvements in behavior. Therefore it was concluded that levetiracetam may be extremely effective in patients with developmental disability. Behavioral abnormalities improved in 50\% of the patients.

\section{Conclusion}

Levetiracetam is a second generation anti epileptics. It is used as adjunctive therapy for partial onset seizures, generalized tonic-clonic seizures and myoclonic seizures in both children and adults. The studies regarding the elevation of serum total bilirubin and hyponatremic condition associated with the treatment of levetiracetam are very rare. The importance of serum total bilirubin elevation and hyponatremic condition with levetiracetam can be magnified by carrying out further studies and assessments. Patients taking levetiracetam should monitor the serum total bilirubin level and serum sodium levels.

\section{References}

Aasim Ali Syed, Christopher, D., Adams. 2012. Acute liver failure following levetiracetam therapy for seizure prophylaxis in traumatic brain injury. Case Reports in Clin. Med., Vol.1 No.2.

Gordon Gibson. 2002. Efficacy of levetiracetam in developmentally disabled patients: a review of the literature and six case reports, Volume 3, Issue 3, Pages 280-284.

Hae Won Shin and Robin Davis. 2013. Review of Levetiracetam as a First Line Treatment in Status Epilepticus in the Adult Patients. Frontiers in Neurol., 4: 111.

Meshkini, A., Ghojazadeh, M., Golbahar-Haghighi, A., Zarea-Gavgani, V., Lotfi-Sadigh, S. 2015. Comparison of levetiracetam versus phenytoin for seizure prophylaxis in patients with traumatic brain injury: A meta-analysis. J. Anal. Res. Clin. Med., 3(2): 118-25.

Neligan, A., Hauser, W.A., Sander, J.W. 2012. "The epidemiology of the epilepsies.". Handbook of Clinical Neurol., 107: 113-33.

Swaroop, H.S., Ananya, C., Nithin, K., Jayashankar, C.A., Satish Babu, H.V., Srinivas, B.N. 2013. Levetiracetam: A Review of its use in the treatment of epilepsy. Int. J. Med. Biomed. Res., Volume 2 Issue 3, 2(3): 166-172. 
Vincenzo Belcastro. Levetiracetam-associated

hyponatremia. Seizure, 17(4): 389-90.
Zimmerman, H.J. 1999. Hepatotoxicity: the adverse effects of drugs and other chemicals on the liver. 2nd ed. Lippincott, pp. 498-516.

\section{How to cite this article:}

Lincy Joseph, Mathew George, Lintu Mariam Joshua. 2017. Estimation of Serum Total Bilirubin Level and Hyponatremic Effect Associated with the Use of Levetiracetam Therapy in Epileptic Patients. Int.J.Curr.Res.Aca.Rev. 5(1), 94-97. doi: $\underline{\text { http://dx.doi.org/10.20546/ijcrar.2017.501.011 }}$ 